\title{
SCALING THE HEIGHTS
}

Cities are magnets for people, resources and infrastructure - essential components for the generation of new ideas. We present here other factors that make for an ideal knowledge city. The elements might seem obvious, but there's no magic formula for how to combine them.

\section{MAPPING}

\section{SCIENCE CITIES}

The 500 cities

plotted on this map account for more than $90 \%$ of the share of authorship in the Nature Index in 2016.

\section{IN A HUDDLE}

Agglomeration is the term used by urban economists to describe the added benefits that come from companies clustering in cities. Spatial proximity also seems to amplify knowledge. The relatively small city of Boston-Cambridge has two of the top 10 academic institutions in the Nature Index 2016, ranked by their weighted fractional count - Harvard University and MIT. China's top two institutions are less than a kilometre apart in Beijing. And King's College London, Imperial College London and UCL form a successful triangle, north of the river Thames.

GLOBAL
CONNECTIONS
While advances in
digital technology
have made it easier for
people to communicate
and collaborate,
meeting face-to-face
is ideal, especially for
serendipitous discovery.
That can mean being
on the next flight to
be with the rest of the
team when something
is about to break. If
they're in Atlanta, so
much the better: the
Megahubs Index ranked
Hartsfield-Jackson
Atlanta International
Airport as the most
connected airport in the
world in 2016 .

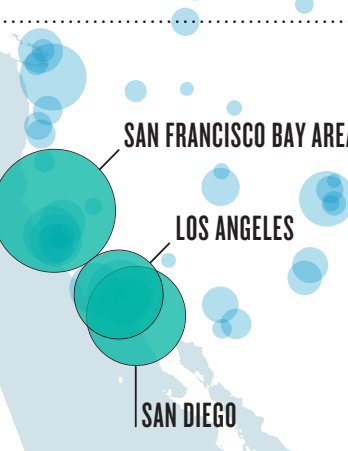

BOSTON-CAMBRIDGE

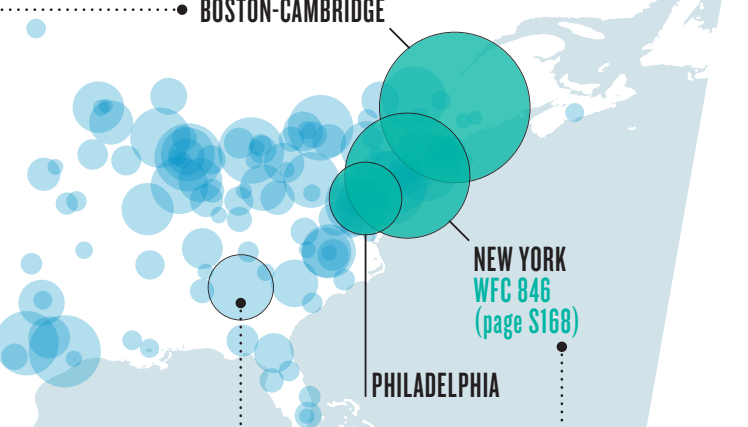

DIVERSITY

Some of the most diverse cities in the world are also scientific powerhouses.

According to the

International Organization of Migration, the United States is the world's top relocation destination, taking more than 40 million immigrants, $85 \%$ of whom have settled in the country's top 100 metropolitan areas. New York is at the head of the queue. A 2000-2010 analysis of patent records from the World Intellectual Property Organization also found that the US had the most foreignborn inventors. Of course, diversity refers not just to population, but to industries and activities too.

Imagine global high-quality research as a skyline where the buildings are cities. 'Uptown', the North American cities of Boston-Cambridge, New York and San Francisco peek above the crowd. In midtown, Paris and London are highest among a dense conglomeration of European towers. But the tallest skyscraper is in downtown Asia, where Beijing is nearly twice the height of nearby Shanghai. 
Bubbles and bars are sized by WFC 2016

Cities along the skyline are positioned based on their longitude. The top 20 cities are shaded teal.

\section{DATA ANALYSIS BY AARON BALLAGH, DATA VISUALIZATION BY DANIEL ORMELLA}

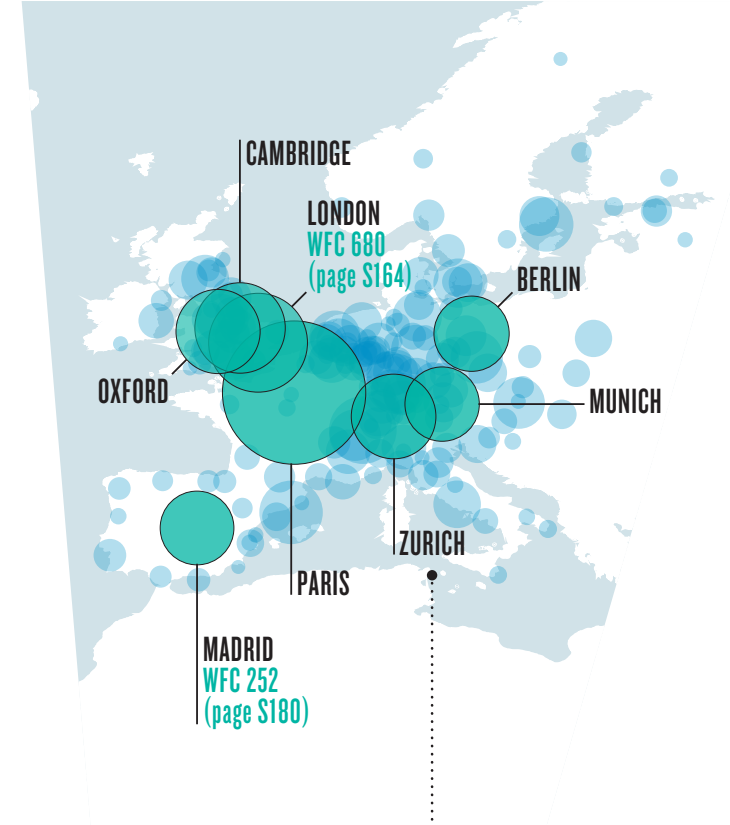

JE NE SAIS QUOI

Work opportunities may be only one factor in a researcher's choice of cities. They might like a city for its clean-energy commitment, such as Zurich, which topped the Sustainable Cities Index in 2016, or choose a city such as Amsterdam for its green spaces and cycle paths. For theatre fans New York's Broadway or London's West End might appeal.

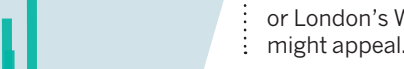

\section{SUPERSIZED}

Larger cities rev up the räte of discovery by creating more opportunities for people with a diverse skillset to meet. They also offer the niche markets needed to service these ideas. "There is nothing magical about cities," says Deborah Strumsky, an urban economist at Arizona State University. "Cities are more productive because they have more inventors per capita - the larger the city, the larger its share of inventors." With a population of more than 36 million, Greater Tokyo is the largest metropolitan area in the world.

\section{DIGITAL ACCESS}

In an ideal knowledge city, researchers can easily communicate with the world, digitally or in person. Seoul offers the fastest home broadband plans for the cheapest price, according to the Open Technology Institute. It also operates the world's largest metropolitan subway system. Ease of communication may have contributed towards researchers in Seoul being granted 22,513 patents from the United States

Patent and Trademark Office in 2016 - the highest for any city.

\section{BEIJING}

WFC 1,693 (page S186)

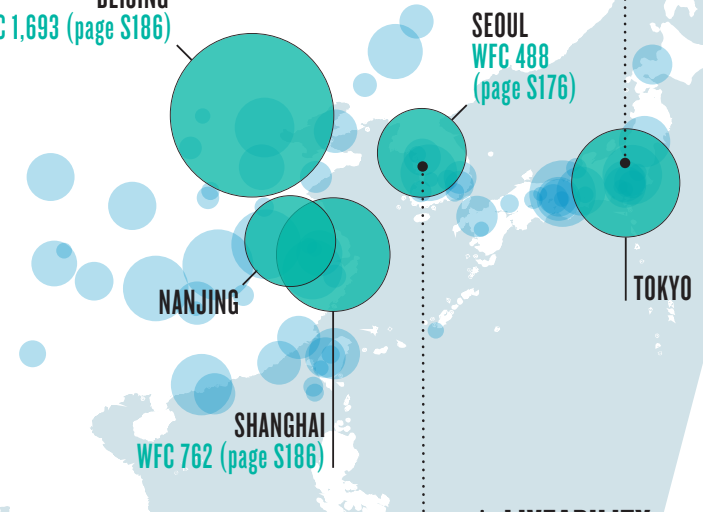

LIVEABILITY

The amenities of big cities can make them expensive, but the most affordable cities might lack qualities that make them attractive to live in. Almaty in Kazakhstan was ranked the cheapest city in the world to live in according to The Economist's Worldwide Cost of Living 2017, though it comes with a measure of economic insecurity. The most 'liveable' city in 2017 was Melbourne Australia taking into account stability, healthcare, culture and environment, education and infrastructure.

Madrid

Oxford
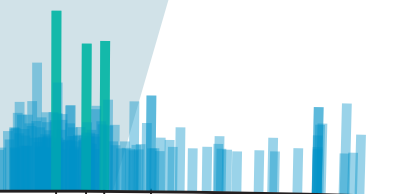

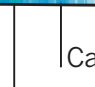

Munich

Zurich

Paris

Cambridge

London
Rehovot
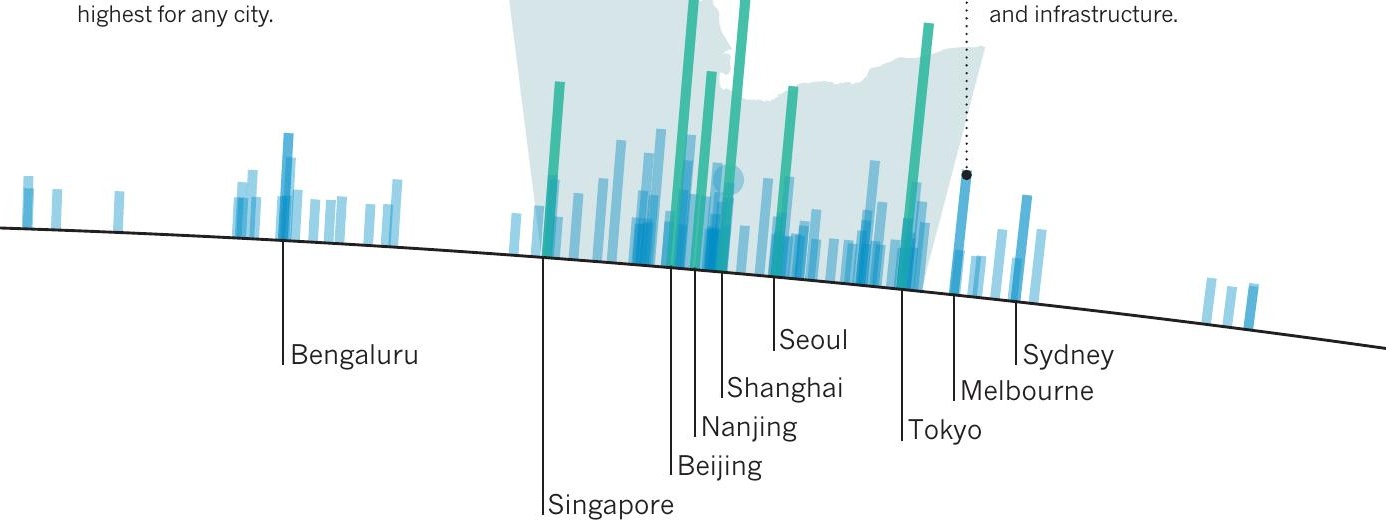